\title{
Long-Term Clinical Effects of Carotid Intraplaque Neovascularization in Patients with Coronary Artery
} Disease

\author{
Hyemoon Chung, MD, PhD, Bu Yong Kim, BN, Hyun Soo Kim, MD, PhD, Hyung Oh Kim, MD, \\ Jung Myung Lee, MD, PhD, Jong Shin Woo, MD, PhD, Jin Bae Kim, MD, PhD, Woo-Shik Kim, MD, PhD, \\ Kwon Sam Kim, MD, PhD, Weon Kim, MD, PhD \\ All authors: Cardiovascular Division, Department of Internal Medicine, Kyung Hee University Medical Center, Kyung Hee University, Seoul, Korea
}

Objective: To investigate the predictive value of intraplaque neovascularization (IPN) for cardiovascular outcomes.

Materials and Methods: We evaluated 217 patients with coronary artery disease (CAD) (158 men; mean age, $68 \pm 10$ years) with a maximal carotid plaque thickness $\geq 1.5 \mathrm{~mm}$ for the presence of IPN using contrast-enhanced ultrasonography. We compared patients with $(n=116)$ and without $(n=101)$ IPN during the follow-up period and investigated the predictors of major adverse cardiovascular events (MACE), including cardiac death, myocardial infarction, coronary artery revascularization, and transient ischemic accident/stroke.

Results: During the mean follow-up period of $995 \pm 610$ days, the MACE rate was $6 \%(13 / 217)$. Patients with IPN had a higher maximal thickness than those without IPN $(2.86 \pm 1.01$ vs. $2.61 \pm 0.84 \mathrm{~mm}, p=0.046)$. Common carotid artery-peak systolic velocity, left ventricular mass index (LVMI), and ventricular-vascular coupling index were significantly correlated with MACE. However, on multivariate Cox regression analysis, increased LVMI was independently related to MACE $(p<0.05)$. The presence of IPN could not predict MACE.

Conclusion: The presence of IPN was related to a higher plaque thickness but could not predict cardiovascular outcomes better than conventional clinical factors in patients with CAD.

Keywords: Carotid intraplaque neovascularization; Contrast-enhanced ultrasound; Cardiovascular outcome

\section{INTRODUCTION}

Carotid intraplaque neovascularization (IPN) is a wellknown major feature of plaque vulnerability (1-3). However,

Received: July 22, 2019 Revised: November 20, 2019

Accepted: February 9, 2020

This research was supported by the National Research Foundation of Republic of Korea (2018R1A2B6009316).

Corresponding author: Weon Kim, MD, PhD, Cardiovascular Division, Department of Internal Medicine, Kyung Hee University Medical Center, Kyung Hee University, 23 Kyungheedae-ro, Dongdaemun-gu, Seoul 02447, Korea.

- Tel: (822) 958-8167 - Fax: (822) 958-8160

- E-mail: mylovekw@hanmail.net

This is an Open Access article distributed under the terms of the Creative Commons Attribution Non-Commercial License (https://creativecommons.org/licenses/by-nc/4.0) which permits unrestricted non-commercial use, distribution, and reproduction in any medium, provided the original work is properly cited. the significant factor contributing to plaque vulnerability remains undetermined (4). Neovascularization is an obvious crucial source of intraplaque hemorrhage that is associated with the progression to plaque rupture. Previous studies demonstrated the association between IPN and patient symptoms (5), cardiovascular risk factors (6), and future stroke events (7). However, large population-based datasets are lacking. Contrast-enhanced ultrasonography (CEUS) is an emerging technique that serves as a valuable method for the early detection of premature atherosclerosis and detection of vulnerable plaques in at-risk populations (8). Carotid plaque contrast agent enhancement is correlated with histological density of neovessels (9). Although carotid IPN is associated with plaque vulnerability, its prognostic value is unclear. Therefore, we evaluated the clinical and hemodynamic characteristics of the presence of IPN using CEUS and investigated the association between 
the presence of IPN and cardiovascular outcomes.

\section{MATERIALS AND METHODS}

\section{Study Population}

This prospective observational study was performed from May 2010 to February 2015 in a single tertiary referral hospital. A total of 217 patients with stable coronary artery disease (CAD) and carotid plaque (thickness $\geq 1.5 \mathrm{~mm}$ ) confirmed by carotid artery ultrasound examination were consecutively enrolled in this study. CEUS was performed in all patients. Written informed consent was obtained from all participants. The exclusion criteria were: 1 ) previous history of percutaneous carotid artery intervention or endarterectomy; 2) significant valvular dysfunction; 3) connective tissue disease; 4) malignancy; and 5) chronic kidney disease characterized by creatinine clearance < $30 \mathrm{~mL} / \mathrm{min}$. This study was performed according to the principles of the Declaration of Helsinki and approved by the local ethics committee (Kyung Hee University Medical Center IRB 1212-07-A2; ClinicalTrials.gov ID, NCT01842490).

\section{Clinical and Conventional Echocardiographic Findings}

Each patient's baseline medical history, blood pressure, electrocardiographic findings, and applanation arterial tonometry findings were collected from their medical records. Flow-mediate dilation (FMD) was measured in 87 patients. From the echocardiographic data, left ventricular (LV) end-diastolic elastance index was calculated as E/ $\mathrm{e}^{\prime}$ /stroke volume, LV end-systolic elastance (Ees) as endsystolic pressure/LV end-systolic volume, effective arterial elastance (Ea) as end-systolic pressure/stroke volume, and ventricular-vascular coupling index (VVI) as Ees/Ea. Here, $E / e^{\prime}$ is the ratio of mitral peak velocity of early filling (E) to early diastolic mitral annular velocity $\left(\mathrm{e}^{\prime}\right)$ and end-systolic pressure is calculated by $(2 \times$ systolic blood pressure + diastolic blood pressure) $/ 3(10,11)$.

\section{Conventional Carotid Doppler Ultrasonography and CEUS}

CEUS was performed using an Acuson Sequoia 512 system (Siemens Healthineers, Munich, Germany) or Vivid E9 (GE Healthcare, Milwaukee, WI, USA) with a $15-\mathrm{MHz}$ imaging transducer equipped with ultrasound contrast software. Image acquisition was performed according to the current guidelines (12). The common carotid artery (CCA), carotid bulb, extracranial internal carotid artery (ICA), and external carotid artery (ECA) were identified. All carotid measurements were performed using semi-automated vessel wall detection software. Following short-axis image acquisition, long-axis B-mode images were acquired for subsequent measurements. Carotid intima-medial thickness (IMT) was measured at the far wall of the CCA at a site approximately $1 \mathrm{~cm}$ proximal to the carotid bulb using an automated edge detection system. A plaque was defined as a protrusion of the vessel wall into the arterial lumen of at least $0.5 \mathrm{~mm}$ with an IMT $>50 \%$ that of the surrounding sites or $>1.5 \mathrm{~mm}$ (12). CEUS analysis of plaques $<1.5 \mathrm{~mm}$ in size showed less positive IPN results in our laboratory; thus, we chose carotid plaques $>1.5 \mathrm{~mm}$ in size to increase the possibility of a definite positive IPN on CEUS. Peak systolic velocity (PSV) and end-diastolic velocity (EDV) were measured in the CCA, ECA, and ICA using a semiautomatic analysis system. The resistive index (RI) was calculated as PSV-EDV/PSV. All CEUS studies were performed using ultrasound contrast agents such as Perflutren lipid microspheres (Definity, Bristol-Myers Squibb Medical Imaging, MA, USA) or phospholipid-stabilized microspheres of sulfur hexafluoride (SonoVue, Bracco Altana Pharma, Germany). Contrast agent was injected via a peripheral vein as a 2-mL bolus, followed by a $5-\mathrm{mL}$ saline flush. After the contrast agent was injected, longitudinal imaging was performed to evaluate the presence of IPN. Representative cases are presented in Figure 1 and Supplementary Movie 1.

\section{Clinical Follow-Up}

Clinical follow-ups were conducted by medical record review or telephone interview. The primary end-point was major adverse cardiovascular event (MACE) defined as cardiac death, acute myocardial infarction (AMI), CAD requiring coronary revascularization, or stroke/transient ischemic attack (TIA).

\section{Statistical Analysis}

Normally distributed continuous variables are reported as mean \pm standard deviation (SD) or $95 \%$ confidence interval (CI). Student's $t$ test was used to compare the means of continuous variables that were approximately normally distributed between the two groups. Normality was determined using the Kolmogorov-Smirnov goodnessof-fit test. Categorical variables are reported as count (percentage) and compared using Fisher's exact test. Independent predictors of MACE were assessed first using univariate Cox proportional hazards regression models and 

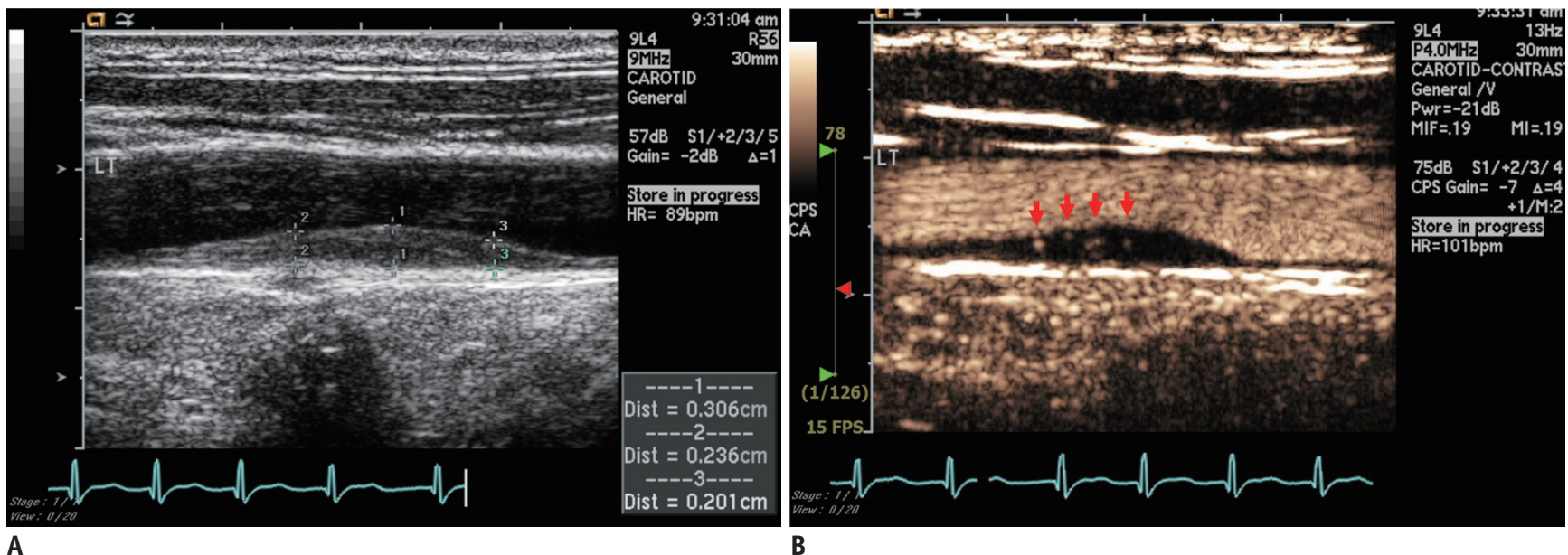

Fig. 1. Representative cases of IPN visualized using contrast-enhanced ultrasound.

Standard ultrasound imaging of CCA (A) and after injection of bolus of contrast agent (B). Bright spots of contrast agent (red arrows) indicate neovessels throughout plaque. $C \mathrm{CA}=$ common carotid artery, $\mathrm{IPN}=$ intraplaque neovascularization

then using multivariable models. The multivariate analysis was performed using variables with $p<0.05$ on univariate analysis. All statistical analyses were performed using SPSS (version 20.0, IBM Corp., Armonk, NY, USA). Two-sided $p$ values $<0.05$ were considered significant.

\section{RESULTS}

\section{Baseline Characteristics according to Presence of IPN}

The patients' baseline characteristics are shown in Table 1. The mean age of the enrolled patients was $68 \pm 10$ years; $59(27.2 \%)$ were women. The presence of hypertension, diabetes, CAD status, atrial fibrillation, smoking history, and lipid profile were similar between the two groups (all $p$ $>0.05)$. There were no significant differences in antiplatelet agent or statin use between the two groups (all $p>0.05$ ). The LV ejection fraction was lower in the IPN than in the no IPN group ( $52.7 \pm 23.5 \%$ vs. $44.9 \pm 29.1 \%$, respectively; $p=$ 0.032). There was no intergroup difference in LV dimensions, left atrial volume index, or right ventricular systolic pressure (all $p>0.05$ ). VVI and central blood pressure also did not differ significantly between the two groups $(p>0.05)$. FMD, representing endothelial function, did not differ between the two groups $(7.6 \pm 6.3 \%$ vs. $5.6 \pm 3.8 \%$ in the with and without IPN groups, respectively; $p=0.089$ ).

\section{Carotid Doppler Characteristics according to Presence of IPN}

The patients' carotid Doppler ultrasound characteristics are described in Table 2. Maximal plaque thickness was higher in the IPN than in the no IPN group $(2.86 \pm 1.01$ $\mathrm{mm}$ vs. $2.61 \pm 0.84 \mathrm{~mm}, p=0.046)$. PSV and EDV of the CCA and ICA did not differ between groups (all $p>0.05$ ). The mean velocities of both carotid arteries were also similar between groups (all $p>0.05$ ). CCA RI was lower in the IPN than in the no IPN group $(0.71 \pm 0.13$ vs. $0.75 \pm$ 0.09 , respectively; $p=0.007$ ).

\section{Clinical Outcomes}

In all patients, the incidence of MACE was $6.0 \%$ (13/217), with a mean follow-up duration of $1015 \pm 598$ days. Among these events, one patient had newly developed AMI, three had unstable angina, five had stable angina requiring coronary revascularization, and four had cerebral infarction or TIA. Among the four patients with cerebral infarction or TIA, one patient was in the no IPN group and three patients were included in the IPN group $(p=0.625)$. No cardiac deaths occurred during follow-up. Univariate Cox regression and Kaplan-Meier analysis revealed that MACE did not differ between the two groups $(6.0 \%[7 / 116]$ vs. $5.9 \%$ [6/101], hazard ratio [HR], 0.996; 95\% CI, 0.335-2.965, $p$ $=0.995$ on the log-rank test) (Table 3, Fig. 2A). Univariate Cox regression analysis revealed that average CCA-PSV was related to MACE (HR, $0.963 ; 95 \% \mathrm{CI}, 0.932-0.995 ; p$ $=0.023)$. VVI was also related to MACE on univariate Cox regression analysis $(p<0.05)$ (Table 3$)$. The 87 patients in whom FMD was measured were further classified into group 1 , with the presence of IPN and decreased FMD (tertile $1,0-3.6 \%$ ), and group 2 (remaining patients). Group 1 was not significantly related to MACE on univariate Cox regression analysis (HR, 0.035; 95\% CI, 0-64.733; $p=$ 0.383). Average CCA-PSV tended to be related to MACE after 
Table 1. Baseline Characteristics according to IPN

\begin{tabular}{|c|c|c|c|}
\hline Characteristics & No IPN Group $(n=101)$ & IPN Group $(n=116)$ & $P$ \\
\hline Age, years & $67 \pm 10$ & $69 \pm 10$ & 0.147 \\
\hline Male (\%) & $70(69.3)$ & $88(75.9)$ & 0.289 \\
\hline $\mathrm{BSA}, \mathrm{m}^{2}$ & $1.71 \pm 0.19$ & $1.71 \pm 0.17$ & 0.867 \\
\hline Hypertension (\%) & $83(82.2)$ & $94(81.0)$ & 0.862 \\
\hline Diabetes (\%) & $40(39.6)$ & $47(40.5)$ & 0.999 \\
\hline History of smoking (\%) & $48(47.5)$ & $39(33.9)$ & 0.052 \\
\hline Initial atrial fibrillation (\%) & $8(7.9)$ & $11(9.5)$ & 0.811 \\
\hline History of stroke (\%) & $15(14.9)$ & $24(20.7)$ & 0.291 \\
\hline CAD $(\%)$ & & & 0.960 \\
\hline Minimal disease & $15(14.9)$ & $20(17.2)$ & \\
\hline 1-vessel disease & $57(56.4)$ & $62(53.4)$ & \\
\hline 2-vessel disease & $15(14.9)$ & $18(15.5)$ & \\
\hline 3-vessel disease & $14(13.9)$ & $16(13.8)$ & \\
\hline History of MI (\%) & $21(20.8)$ & $18(15.5)$ & 0.376 \\
\hline $\mathrm{SBP}, \mathrm{mm} \mathrm{Hg}$ & $121 \pm 30$ & $126 \pm 23$ & 0.166 \\
\hline $\mathrm{DBP}, \mathrm{mm} \mathrm{Hg}$ & $70 \pm 19$ & $75 \pm 14$ & 0.050 \\
\hline Heart rate, bpm & $68 \pm 19$ & $70 \pm 19$ & 0.610 \\
\hline Total cholesterol, mg/dL & $170.3 \pm 47.4$ & $160.6 \pm 64.6$ & 0.214 \\
\hline Triglyceride, $\mathrm{mg} / \mathrm{dL}$ & $123.1 \pm 82.6$ & $121.0 \pm 94.5$ & 0.861 \\
\hline HDL cholesterol, mg/dL & $40.3 \pm 17.8$ & $41.0 \pm 18.9$ & 0.785 \\
\hline LDL cholesterol, mg/dL & $99.4 \pm 46.1$ & $94.5 \pm 54.7$ & 0.470 \\
\hline \multicolumn{4}{|l|}{ Medication (\%) } \\
\hline Aspirin & $49(48.5)$ & $50(43.1)$ & 0.495 \\
\hline Clopidogrel & $28(27.7)$ & $38(32.8)$ & 0.461 \\
\hline Cilostazol & $1(1.0)$ & $2(1.7)$ & $>0.999$ \\
\hline DAPT & $16(15.8)$ & $23(19.8)$ & 0.482 \\
\hline Anticoagulation & 0 & $4(3.4)$ & 0.125 \\
\hline Statin & $44(43.6)$ & $58(50.0)$ & 0.413 \\
\hline Intensity of statin & & & 0.760 \\
\hline Low-intensity & $2(2.0)$ & $3(2.6)$ & \\
\hline Moderate-intensity & $40(39.6)$ & $51(44.0)$ & \\
\hline High-intensity & $2(2.0)$ & $4(3.4)$ & \\
\hline \multicolumn{4}{|c|}{ Echocardiographic characteristics* } \\
\hline LV EDD, mm & $42.5 \pm 18.5$ & $41.9 \pm 18.6$ & 0.801 \\
\hline LV ESD, mm & $27.6 \pm 13.3$ & $26.9 \pm 13.0$ & 0.676 \\
\hline LV mass index, $\mathrm{g} / \mathrm{m}^{2}$ & $86.3 \pm 54.3$ & $83.8 \pm 49.7$ & 0.724 \\
\hline LV EF, \% & $52.7 \pm 23.5$ & $44.9 \pm 29.1$ & 0.032 \\
\hline LAVI, $\mathrm{mL} / \mathrm{m}^{2}$ & $24.8 \pm 13.7$ & $26.4 \pm 9.9$ & 0.395 \\
\hline RVSP, mm Hg & $26.0 \pm 5.9$ & $24.6 \pm 6.9$ & 0.320 \\
\hline$E / e^{\prime}$ & $12.5 \pm 3.9$ & $12.1 \pm 5.1$ & 0.571 \\
\hline Ed & $0.22 \pm 0.15$ & $0.22 \pm 0.14$ & 0.935 \\
\hline Ees & $11.00 \pm 14.47$ & $9.64 \pm 4.62$ & 0.390 \\
\hline VVI & $2.17 \pm 1.20$ & $2.28 \pm 1.72$ & 0.618 \\
\hline $\mathrm{cSBP}, \mathrm{mm} \mathrm{Hg}$ * & $137 \pm 28$ & $140 \pm 26$ & 0.471 \\
\hline $\mathrm{cDBP}, \mathrm{mm} \mathrm{Hg}$ * & $73 \pm 16$ & $74 \pm 11$ & 0.823 \\
\hline $\mathrm{AI}, \%^{\dagger}$ & $82.2 \pm 18.2$ & $81.7 \pm 12.9$ & 0.842 \\
\hline AI $75 \%{ }^{\dagger}$ & $79.1 \pm 16.7$ & $79.0 \pm 12.1$ & 0.966 \\
\hline FMD, \% ${ }^{\ddagger}$ & $5.6 \pm 3.8$ & $7.6 \pm 6.3$ & 0.089 \\
\hline
\end{tabular}

*Analysis of 174 patients for whom transthoracic echocardiography was performed, ${ }^{\dagger}$ Analysis of 146 patients for whom cSBP was measured; 85 and 61 patients with or without IPN, respectively, ${ }^{\ddagger}$ Analysis of 87 patients for whom FMD was measured; 50 and 37 patients with or without IPN, respectively. $\mathrm{AI}=$ augmentation index, $\mathrm{AI} 75 \%=$ heart rate corrected $\mathrm{AI}, \mathrm{BSA}=$ body surface area, $\mathrm{CAD}=\mathrm{coronary}$ artery disease, $\mathrm{CDBP}=$ central diastolic blood pressure, $\mathrm{CSBP}=$ central systolic blood pressure, $\mathrm{DAPT}=$ dual antiplatelet therapy, $\mathrm{DBP}=$ diastolic blood pressure, $E d=$ end-diastolic elastance, $E D D=$ end-diastolic dimension, Ees = end-systolic elastance, $E F=$ ejection fraction, $\mathrm{ESD}=$ end-systolic dimension, $\mathrm{E} / \mathrm{e}^{\prime}=$ ratio of mitral peak velocity of early filling (E) to early diastolic mitral annular velocity ( $\left.\mathrm{e}^{\prime}\right)$, FMD = flow-mediate dilation, $\mathrm{HDL}=$ high-density lipoprotein, $\mathrm{IPN}=$ intraplaque neovascularization, $\mathrm{LAVI}=$ left atrial volume index, $\mathrm{LDL}=$ low-density lipoprotein, $\mathrm{LV}=$ left ventricular, $\mathrm{MI}=$ myocardial infarction, RVSP = right ventricular systolic pressure, SBP = systolic blood pressure, VVI = ventricular-vascular coupling index 
Table 2. Carotid Ultrasonographic Characteristics according to IPN

\begin{tabular}{lccc}
\hline \multicolumn{1}{c}{ Characteristics } & No IPN Group $(\mathrm{n}=101)$ & IPN Group $(\mathrm{n}=116)$ & $P$ \\
\hline Maximal thickness of plaque, $\mathrm{mm}$ & $2.61 \pm 0.84$ & $2.86 \pm 1.01$ & 0.046 \\
Left CCA PSV, cm/s & $51.10 \pm 18.76$ & $52.19 \pm 21.78$ & 0.932 \\
Right CCA PSV, cm/s & $49.71 \pm 20.26$ & $48.40 \pm 22.43$ & 0.821 \\
Average CCA PSV, cm/s & $48.38 \pm 18.69$ & $48.88 \pm 21.52$ & 0.858 \\
Left CCA EDV, cm/s & $12.21 \pm 6.11$ & $13.79 \pm 7.36$ & 0.906 \\
Right CCA EDV, cm/s & $12.02 \pm 6.43$ & $12.88 \pm 6.57$ & 0.767 \\
Average CCA EDV, cm/s & $11.63 \pm 6.03$ & $13.04 \pm 6.62$ & 0.107 \\
Left ICA PSV, cm/s & $59.15 \pm 34.72$ & $66.87 \pm 51.59$ & \\
Right ICA PSV, cm/s & $65.61 \pm 60.79$ & $55.56 \pm 26.00$ & \\
Average ICA PSV, cm/s & $60.75 \pm 39.23$ & $60.17 \pm 33.37$ & 0.361 \\
Left ICA EDV, cm/s & $19.28 \pm 11.99$ & $23.23 \pm 18.95$ & 0.167 \\
Right ICA EDV, cm/s & $21.72 \pm 19.49$ & $19.65 \pm 10.87$ & 0.908 \\
Average ICA EDV, cm/s & $19.76 \pm 13.53$ & $21.06 \pm 12.50$ & 0.542 \\
Average RI of CCA & $0.75 \pm 0.09$ & $0.71 \pm 0.13$ & 0.471 \\
\hline
\end{tabular}

$\mathrm{CCA}=$ common carotid artery, EDV $=$ end-diastolic velocity, ICA = internal carotid artery, PSV = peak systolic velocity, RI = resistive index

Table 3. Univariate Cox Regression Analysis for Predictors of MACE

\begin{tabular}{|c|c|c|c|}
\hline Characteristics & HR & $95 \%$ CI & $P$ \\
\hline IPN & 0.996 & $0.335-2.965$ & 0.995 \\
\hline Maximal plaque thickness, per $\mathrm{mm}$ & 1.117 & $0.684-1.824$ & 0.659 \\
\hline Age, per year & 1.012 & $0.956-1.071$ & 0.694 \\
\hline Male & 3.961 & $0.515-30.478$ & 0.186 \\
\hline Initial atrial fibrillation & 3.515 & $0.749-16.486$ & 0.111 \\
\hline Diabetes & 0.308 & $0.068-1.389$ & 0.125 \\
\hline Hypertension & 1.050 & $0.232-4.754$ & 0.950 \\
\hline Smoking & 1.261 & $0.424-3.754$ & 0.676 \\
\hline Average CCA PSV, per $\mathrm{cm} / \mathrm{s}$ & 0.963 & $0.933-0.995$ & 0.023 \\
\hline Average CCA EDV, per $\mathrm{cm} / \mathrm{s}$ & 0.940 & $0.855-1.033$ & 0.199 \\
\hline Average ICA PSV, per cm/s & 0.994 & $0.976-1.012$ & 0.503 \\
\hline Average ICA EDV, per $\mathrm{cm} / \mathrm{s}$ & 0.990 & $0.948-1.033$ & 0.635 \\
\hline $\mathrm{EF}$, per $\%$ & 1.001 & $0.981-1.022$ & 0.899 \\
\hline LV mass index, per $\mathrm{g} / \mathrm{m}^{2}$ & 1.016 & $1.000-1.032$ & 0.054 \\
\hline LAVI, $\mathrm{mL} / \mathrm{m}^{2}$ & 1.016 & $0.972-1.061$ & 0.494 \\
\hline RVSP, per mm Hg & 0.971 & $0.874-1.079$ & 0.587 \\
\hline$E / e^{\prime}$ & 1.056 & $0.933-1.196$ & 0.388 \\
\hline $\mathrm{Ed}$ & 6.850 & $0.317-147.848$ & 0.220 \\
\hline Ees & 1.005 & $0.951-1.062$ & 0.853 \\
\hline Ea & 1.747 & $0.876-3.484$ & 0.113 \\
\hline VVI & 1.366 & $1.029-1.815$ & 0.031 \\
\hline cSBP, per mm Hg* & 1.009 & $0.983-1.036$ & 0.490 \\
\hline cDBP, per $\mathrm{mm} \mathrm{Hg}$ * & 1.028 & $0.981-1.078$ & 0.246 \\
\hline AI $75 \% *$ & 0.964 & $0.930-1.000$ & 0.050 \\
\hline FMD, per $\%^{\dagger}$ & 0.988 & $0.859-1.123$ & 0.854 \\
\hline Group $1^{\ddagger}$ & 0.035 & 0-64.733 & 0.383 \\
\hline
\end{tabular}

*Analysis of 146 patients for whom cSBP was measured; 85 and 61 patients with or without IPN, respectively, ${ }^{\dagger}$ Analysis of 87 patients for whom FMD was measured; 50 and 37 patients with or without IPN, respectively, ${ }^{\ddagger}$ Among 87 patients for whom FMD was measured, we further classified patients into two groups; group 1, presence of IPN and decreased FMD (tertile 1, 0-3.6\%) vs. group 2 (remained patients). $\mathrm{CI}=$ confidence interval, $\mathrm{Ea}=$ effective arterial elastance, $\mathrm{HR}=$ hazard ratio, $\mathrm{MACE}=$ major adverse cardiovascular event multivariate Cox regression analysis, but this association was not statistically significant $(p=0.065)$ (Table 4). Figure 2B shows the Kaplan-Meier curve for MACE according to the average CCA-PSV (log rank $p=0.058)$.

The optimal cutoff value for average CCA-PSV was 36.8 $\mathrm{cm} / \mathrm{s}(38.5 \%$ sensitivity and $70.4 \%$ specificity) and the area under the receiver operating characteristic curve of the mean PSV of the CCA for MACE was 0.274 (95\% CI, 0.132$0.416 ; p=0.006)$.

\section{DISCUSSION}

Inflammation and neovascularization are two features known to indicate plaque vulnerability (1). Here we investigated the effect of IPN on the clinical outcomes of patients with carotid plaques. There are two major aspects to our study. The first is that we investigated the predictive value of IPN during a longer follow-up period, while the second is that we evaluated echocardiographic and hemodynamic characteristics, in addition to performing carotid artery ultrasound examinations. In our previous study, the presence of IPN was not related to 1-year cardiovascular outcome but was related to matrix metalloproteinase-9, a key protease in ruptured plaques (13). Although we extended the follow-up period, the result was similar to that of our previous report; the presence of IPN was also not related to long-term cardiovascular outcomes in patients with carotid plaques.

\section{Contribution of Neovascularization to Cardiovascular Events}

The association between neovascularization, 


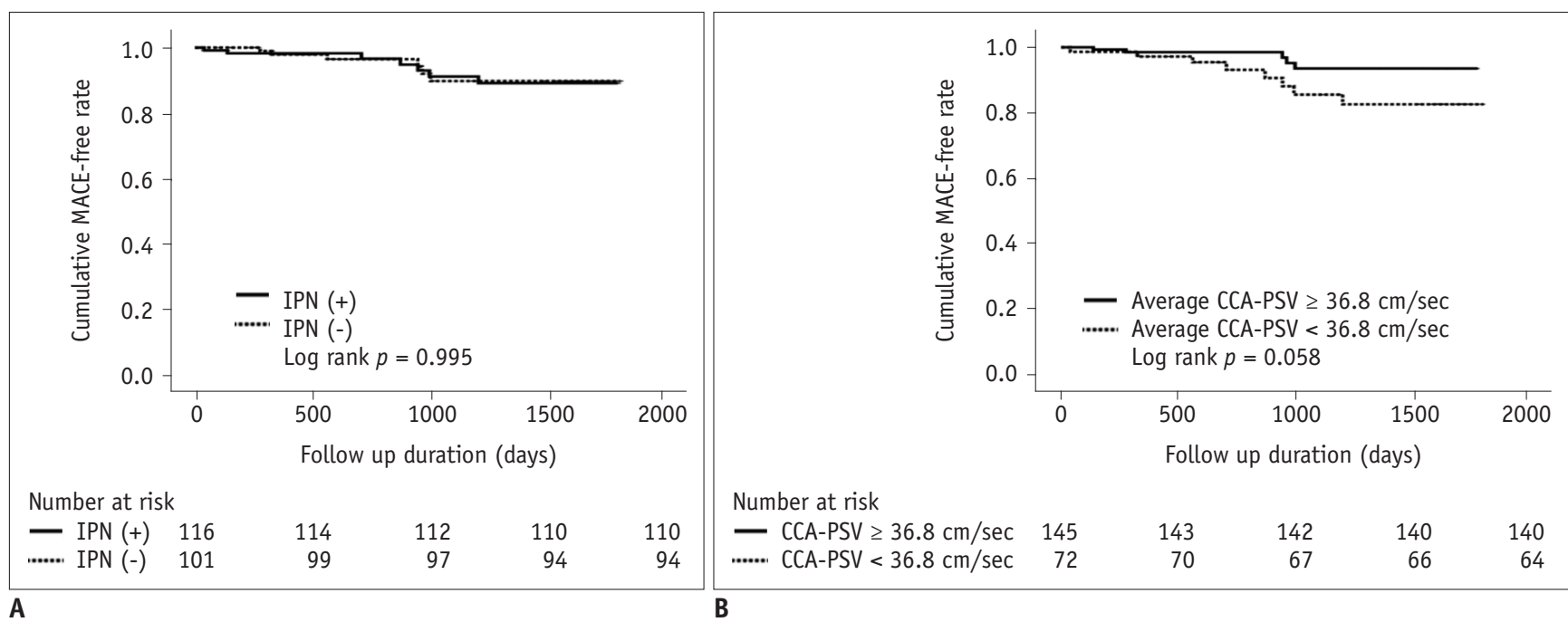

Fig. 2. Kaplan-Meier survival curves for MACE in patients according to presence of IPN $(A)$ and average CCA-PSV $(B)$. MACE $=$ major adverse cardiovascular event, PSV = peak systolic velocity

Table 4. Multivariate Cox Regression Analysis for Predictors of MACE

\begin{tabular}{lccc}
\hline \multicolumn{1}{c}{ Characteristics } & HR & $95 \%$ CI & $P$ \\
\hline Age, per year & 0.988 & $0.929-1.051$ & 0.700 \\
Average CCA PSV, per cm/s & 0.966 & $0.932-1.002$ & 0.065 \\
VVI & 1.149 & $0.916-1.702$ & 0.160 \\
\hline
\end{tabular}

inflammation, and plaque vulnerability has been reported by several studies $(14,15)$. Willems et al. (16) reported that the number of mast cells was associated with plaque microvessel density and that the presence of mast cells was associated with adverse cardiovascular events. Vascular inflammation is a driver of plaque formation, typical feature of plaque rupture, and leading factor for cardiovascular events (17). We believe that the discrepancy between IPN and clinical outcomes may be because of two reasons. First, the characteristics of neovascularization and inflammation differ. Neovascularization is a local process, whereas inflammation is a systemic process affecting the systemic coagulation pathway, as well as local processes in the plaque (4). Second, the intravascular distribution of contrast agent may contribute to the discrepancy. Demeure et al. (9) reported that inflammation and IPN were not systemically associated with carotid plaque. CEUS reflects neovascularization but not endothelial permeability because of the intravascular characteristics of the contrast agent, as described by Demeure et al (9). Endothelial permeability is an important part of inflammation, and CEUS does not completely represent inflammation because it cannot bridge the endothelial barrier. In addition, histologically proven neovascularization and IPN using CEUS are not always consistent, especially in cases of mild IPN $(9,18)$.

Several studies indicated the predictive value of IPN for cardiovascular outcomes of patients with overt cardiovascular diseases $(1,7)$. However, there are also conflicting data about whether IPN may be associated with clinical characteristics and outcomes. Van den 0ord et al. (19) found no association between amount of IPN and the presence of complications in patients with asymptomatic diabetes. Although neovascularization is a crucial source of intraplaque hemorrhage associated with the progression to plaque rupture, not all neovascularization leads directly to plaque vulnerability. We think that might be related with conflicting data regarding whether IPN is associated with adverse clinical outcomes. Plaques with neovascularization may be more vulnerable when combined with active inflammation. In our study cohort, the presence of IPN did not predict cardiovascular outcomes. We enrolled patients with subclinical disease and those at overt cardiovascular risk. In these patients, heterogeneous inflammatory status might be associated with variable clinical outcomes. We also analyzed FMD to assess endothelial function, which reflects inflammatory status. In this study, the presence of IPN was unrelated to that of FMD and the presence of IPN and decreased FMD was not a predictor of MACE. Despite the lack of significance, a lower FMD and presence of IPN had an inverse relationship ( $7.6 \pm 6.3 \%$ vs. $5.6 \pm 3.8 \%$ in the with and without IPN groups, respectively, $p=0.089$ ). One study reported a weak inverse relationship between neovascularization on dynamic contrast-enhanced magnetic 
resonance imaging and inflammation on ${ }^{18} \mathrm{~F}$-fluorodeoxyglucose positron emission tomography (20). This result might be related to various compounding factors. Neovascularization and inflammation reflect different stages of plaque progression and provide complementary information. This point may also explain our result.

Moreover, the association between IPN and stroke has been reported previously (21). In our study, cerebrovascular events occurred in four patients, one in the without IPN group and three in the with IPN group. Due to the small number of events, there was no statistical significance.

\section{Predictors of Cardiovascular Outcomes}

In our study, IPN did not predict future cardiovascular events. Although the statistical significance disappeared after the adjustment for these factors, the PSV of the CCA was associated with cardiovascular outcomes. Hemodynamic indices, such as the VVI, were also associated with MACE, although they were also non-significant after multivariate analysis.

\section{Limitation}

The present study has several limitations. First, it was performed in a single tertiary center and included a small number of patients. Therefore, we cannot exclude selection and referral bias. Second, the study population was small and the population had heterogeneous conditions and various atherosclerotic burdens. Therefore, we think it would be difficult to generalize our results. Third, IPN grading was not performed because of a lack of established guidelines. Third, a high SD of the follow-up period may have been a source of bias. Finally, inconsistent use of contrast agent might have affected our results.

In conclusion, neovascularization may be a critical source of plaque rupture and subsequent adverse cardiocerebrovascular events. Neovascularization does not directly reflect inflammation, but it is a key feature in plaque vulnerability and can offer crucial information regarding atherosclerotic status. In addition, CEUS cannot reflect endothelial permeability, and its results were not completely consistent with the histological findings. Although the predictive value of CEUS was not demonstrated here, its combination with inflammatory factors may be informative in a patient's cardiovascular course. Further studies may identify predictors of future vascular events in patients with neovascularization within carotid plaques.

\section{Supplementary Materials}

The Data Supplement is available with this article at https://doi.org/10.3348/kjr.2019.0550.

\section{Supplementary Movie Legends}

Movie 1. Representative contrast-enhanced ultrasonography (A) with and (B) without intraplaque neovascularization.

\section{Conflicts of Interest}

The authors have no potential conflicts of interest to disclose.

\section{Acknowledgments}

We thank the study participants for making this study possible.

$$
\begin{aligned}
& \text { ORCID iDs } \\
& \text { Weon Kim } \\
& \text { https://orcid.org/0000-0003-1264-9870 } \\
& \text { Hyemoon Chung } \\
& \text { https://orcid.org/0000-0002-5615-6245 } \\
& \text { Hyung Oh Kim } \\
& \text { https://orcid.org/0000-0003-2157-441X } \\
& \text { Jung Myung Lee } \\
& \text { https://orcid.org/0000-0002-1904-5335 } \\
& \text { Jong Shin Woo } \\
& \text { https://orcid.org/0000-0003-0535-1742 } \\
& \text { Jin Bae Kim } \\
& \text { https://orcid.org/0000-0002-0293-0901 } \\
& \text { Woo-Shik Kim } \\
& \text { https://orcid.org/0000-0002-3345-1137 }
\end{aligned}
$$

\section{REFERENCES}

1. Hellings WE, Peeters W, Moll FL, Piers SR, van Setten J, Van der Spek PJ, et al. Composition of carotid atherosclerotic plaque is associated with cardiovascular outcome: a prognostic study. Circulation 2010;121:1941-1950

2. Handa N, Matsumoto M, Maeda H, Hougaku H, Kamada T. Ischemic stroke events and carotid atherosclerosis. Results of the Osaka follow-up study for ultrasonographic assessment of carotid atherosclerosis (the OSACA Study). Stroke 1995;26:1781-1786

3. Doyle B, Caplice N. Plaque neovascularization and antiangiogenic therapy for atherosclerosis. J Am Coll Cardiol 
2007; 49:2073-2080

4. Naghavi M, Libby P, Falk E, Casscells SW, Litovsky S, Rumberger J, et al. From vulnerable plaque to vulnerable patient: a call for new definitions and risk assessment strategies: part I. Circulation 2003;108:1664-1672

5. Owen DR, Shalhoub J, Miller S, Gauthier T, Doryforou 0, Davies $\mathrm{AH}$, et al. Inflammation within carotid atherosclerotic plaque: assessment with late-phase contrast-enhanced US. Radiology 2010;255:638-644

6. Magnoni M, Ammirati E, Moroni F, Norata GD, Camici PG. Impact of cardiovascular risk factors and pharmacologic treatments on carotid intraplaque neovascularization detected by contrast-enhanced ultrasound. J Am Soc Echocardiogr 2019;32:113-120.e6

7. Staub D, Patel MB, Tibrewala A, Ludden D, Johnson M, Espinosa $P$, et al. Vasa vasorum and plaque neovascularization on contrast-enhanced carotid ultrasound imaging correlates with cardiovascular disease and past cardiovascular events. Stroke 2010;41:41-47

8. Staub D, Schinkel AF, Coll B, Coli S, van der Steen AF, Reed $J \mathrm{D}$, et al. Contrast-enhanced ultrasound imaging of the vasa vasorum: from early atherosclerosis to the identification of unstable plaques. JACC Cardiovasc Imaging 2010;3:761-771

9. Demeure F, Bouzin C, Roelants V, Bol A, Verhelst R, Astarci $P$, et al. Head-to-head comparison of inflammation and neovascularization in human carotid plaques: implications for the imaging of vulnerable plaques. Circ Cardiovasc Imaging 2017;10. pii: e005846

10. Her AY, Kim JY, Choi EY, Kim SA, Jae RS, Shim CY, et al. Value of ventricular stiffness index and ventriculoarterial interaction in patients with nonischemic dilated cardiomyopathy. Circ $J$ $2009 ; 73: 1683-1690$

11. Choi EY, Choi BW, Kim SA, Rhee SJ, Shim CY, Kim YJ, et al. Patterns of late gadolinium enhancement are associated with ventricular stiffness in patients with advanced non-ischaemic dilated cardiomyopathy. Eur J Heart Fail 2009;11:573-580

12. Stein JH, Korcarz CE, Hurst RT, Lonn E, Kendall CB, Mohler ER, et al. Use of carotid ultrasound to identify subclinical vascular disease and evaluate cardiovascular disease risk: a consensus statement from the American Society of Echocardiography carotid intima-media thickness task force. Endorsed by the society for vascular medicine. J Am Soc Echocardiogr
2008;21:93-111; quiz 189-190

13. Kim HS, Woo JS, Kim BY, Jang HH, Hwang SJ, Kwon SJ, et al. Biochemical and clinical correlation of intraplaque neovascularization using contrast-enhanced ultrasound of the carotid artery. Atherosclerosis 2014;233:579-583

14. Pelisek J, Well G, Reeps C, Rudelius M, Kuehnl A, Culmes M, et al. Neovascularization and angiogenic factors in advanced human carotid artery stenosis. Circ J 2012;76:1274-1282

15. Purushothaman KR, Purushothaman M, Muntner $P$, Lento PA, O'Connor WN, Sharma SK, et al. Inflammation, neovascularization and intra-plaque hemorrhage are associated with increased reparative collagen content: implication for plaque progression in diabetic atherosclerosis. Vasc Med 2011;16:103-108

16. Willems S, Vink A, Bot I, Quax PH, de Borst GJ, de Vries JP, et al. Mast cells in human carotid atherosclerotic plaques are associated with intraplaque microvessel density and the occurrence of future cardiovascular events. Eur Heart $\mathrm{J}$ 2013;34:3699-3706

17. Ross R. Atherosclerosis--an inflammatory disease. N Engl J Med 1999;340:115-126

18. D'0ria M, Chiarandini S, Pipitone MD, Fisicaro M, Calvagna C, Bussani R, et al. Contrast enhanced ultrasound (CEUS) is not able to identify vulnerable plaques in asymptomatic carotid atherosclerotic disease. Eur J Vasc Endovasc Surg 2018;56:632-642

19. van den Oord SC, Akkus Z, Renaud G, Bosch JG, van der Steen $A F$, Sijbrands EJ, et al. Assessment of carotid atherosclerosis, intraplaque neovascularization, and plaque ulceration using quantitative contrast-enhanced ultrasound in asymptomatic patients with diabetes mellitus. Eur Heart J Cardiovasc Imaging 2014;15:1213-1218

20. Calcagno C, Ramachandran S, Izquierdo-Garcia D, Mani V, Millon A, Rosenbaum D, et al. The complementary roles of dynamic contrast-enhanced MRI and ${ }^{18} \mathrm{~F}$-fluorodeoxyglucose PET/CT for imaging of carotid atherosclerosis. Eur J Nucl Med Mol Imaging 2013;40:1884-1893

21. Song Y, Dang Y, Dang LL, Zhao C, Zheng J, Feng J, et al. Association between intraplaque neovascularization assessed by contrast-enhanced ultrasound and the risk of stroke. Clin Radiol 2020;75:70-75 